\title{
IMAGES OF ISOGENY CLASSES ON MODULAR ELLIPTIC CURVES
}

\author{
FLORIAN BREUER
}

\begin{abstract}
Let $K$ be a number field and $E / K$ a modular elliptic curve, with modular parametrization $\pi: X_{0}(N) \longrightarrow E$ defined over $K$. The purpose of this note is to study the images in $E$ of classes of isogenous points in $X_{0}(N)$.
\end{abstract}

Let $\pi: X_{0}(N) \rightarrow E$ be as above, and denote by $\bar{K}$ an algebraic closure of $K$.

Theorem 1. Let $S \subset X_{0}(N)(\bar{K})$ be an infinite set of points corresponding to elliptic curves which all lie in one isogeny class, but which are not isogenous to $E$ itself. Then the subgroup of $E(\bar{K})$ generated by $\pi(S)$ has infinite rank and finite torsion.

Proof. Write $S=\left\{x_{0}, x_{1}, \ldots\right\}$ and $y_{i}:=\pi\left(x_{i}\right) \in E(\bar{K})$ for $i \geq 0$. We first show that $\langle\pi(S)\rangle$ is not finitely generated, and then that it has finite torsion.

Suppose that $\langle\pi(S)\rangle$ is finitely generated. Then $\langle\pi(S)\rangle \subset E(L)$ for some number field $L$, which we may extend to include $K$. Now $G_{L}:=\operatorname{Gal}(\bar{L} / L)$ acts on each fibre $\pi^{-1}\left(y_{i}\right)$, from which follows that

$$
\left|G_{L} \cdot x_{i}\right| \leq \operatorname{deg}(\pi), \quad \forall i \geq 0
$$

Denote by $E_{i}$ the elliptic curve corresponding to $x_{i}$ for each $i \geq 0$. It is isogenous to $E_{0}$. We now consider two cases.

(i) If $E_{0}$ has complex multiplication, then each $\operatorname{End}\left(E_{i}\right)$ is an order of conductor $f_{i}$ in a fixed quadratic imaginary field $F$. We denote by $h_{F}$ the class number of $F$. Then we have

$$
\begin{aligned}
\left|G_{L} \cdot x_{i}\right| & \geq\left|\operatorname{Pic}\left(\operatorname{End}\left(E_{i}\right)\right)\right| / 2[L: \mathbb{Q}] \quad(\text { by }[2, \text { Chap 10, Theorem 5]) } \\
& \geq \frac{h_{F}}{12[L: \mathbb{Q}]} \cdot f_{i} \prod_{p \mid f_{i}}\left(1-\frac{1}{p}\right) \quad(\text { by }[2, \text { Chap } 8, \text { Theorem } 7]),
\end{aligned}
$$

which tends to $\infty$ as $i \rightarrow \infty$, thus contradicting (1).

(ii) Now suppose that $E_{0}$ does not have complex multiplication. We may write $E_{i}=E_{0} / C_{i}$, with $C_{i} \subset E_{0}$ a cyclic subgroup of order $n_{i}$. Consider the Galois representations

$$
\rho_{n_{i}}: G_{L} \longrightarrow \operatorname{Aut}\left(E_{0}\left[n_{i}\right]\right) \cong \mathrm{GL}_{2}\left(\mathbb{Z} / n_{i} \mathbb{Z}\right)
$$

Received April 5, 2004.

The author would like to thank the Max-Planck-Institut für Mathematik, Bonn, where this paper was written. 
attached to $E_{0}$. From [5, Théorème $\left.3^{\prime}\right]$ follows that there exists a constant $d_{0}$, depending only on $E_{0}$ and on $L$, such that the image of $\rho_{n_{i}}$ has index at most $d_{0}$. Thus

$$
\left|G_{L} \cdot x_{i}\right| \geq\left|\operatorname{Aut}\left(E_{0}\left[n_{i}\right]\right) \cdot C_{i}\right| / d_{0}=\psi\left(n_{i}\right) / d_{0},
$$

where $\psi\left(n_{i}\right)=n_{i} \prod_{p \mid n_{i}}(1+1 / p) \geq n_{i}$ is the number of cyclic subgroups of order $n_{i}$ in $E_{0}\left[n_{i}\right]$. This again contradicts (1), and it follows that $\langle\pi(S)\rangle$ is not finitely generated. Notice that at this point we have not yet used the assumption that the $E_{i}$ are not isogenous to $E$ itself.

We now show that $\langle\pi(S)\rangle$ has finite torsion. Let $K_{0} \supset K$ be a number field over which $E_{0}$ is defined, then every $E_{i}$ is defined over $L_{0}=K_{0}\left(E_{0, \text { tors }}\right)$. From the Weil pairing follows that $K_{0}\left(\mu_{\infty}\right) \subset L_{0}$. From [5, Théorème $\left.6^{\prime \prime \prime}\right]$ and [1, Satz 4] follows that $L_{0} \cap K_{0}\left(E_{\text {tors }}\right)$ is a finite extension of $K_{0}\left(\mu_{\infty}\right)$, as $E$ and $E_{0}$ are not isogenous. Therefore we may write $L_{0} \cap K_{0}\left(E_{\text {tors }}\right)=L\left(\mu_{\infty}\right)$ for some number field $L$. Now from [4] follows that $E_{\text {tors }}\left(L\left(\mu_{\infty}\right)\right)$ is finite, yet $\langle\pi(S)\rangle_{\text {tors }} \subset E_{\text {tors }}\left(L\left(\mu_{\infty}\right)\right)$, which completes our proof.

What happens with images of points isogenous to $E$ itself? Here it is conceivable that the image has infinite torsion, but the following result shows that if $S$ contains an infinite chain of cyclic $m$-isogenies $x_{1} \stackrel{m}{\rightarrow} x_{2} \stackrel{m}{\rightarrow} \cdots$ for $m$ sufficiently large, then infinitely many of the $\pi\left(x_{i}\right)$ 's must be points of infinite order.

Theorem 2. Let $m \geq \max (2, \operatorname{deg}(\pi))$. Then there exist only finitely many pairs of torsion points $y_{1}, y_{2} \in E_{\text {tors }}(\mathbb{C})$ which possess preimages $x_{1} \in \pi^{-1}\left(y_{1}\right)$ and $x_{2} \in \pi^{-1}\left(y_{2}\right)$ corresponding to elliptic curves $E_{1}$ and $E_{2}$ linked by a cyclic isogeny of degree $m$.

Proof. Denote by $T_{m} \subset X_{0}(N) \times X_{0}(N)$ the Hecke correspondence of level $m$, and let $C_{m} \subset E \times E$ denote its image under $\pi \times \pi$. We view $C_{m}$ as a symmetrical correspondence on $E$.

Suppose that $C_{m}$ contains infinitely many torsion points of the abelian variety $A=E \times E$. Then it follows from the Manin-Mumford Conjecture, proved by Raynaud (see [3] for the relevant case), that $C_{m}$ is the translate by a torsion point of an abelian subvariety of $A$. Now, the one-dimensional abelian subvarieties of $A$ are of the form $\{0\} \times E, E \times\{0\}$, or graphs of endomorphisms of $E$. But $C_{m}$ is symmetrical, hence it is a translate of the graph of an automorphism of $E$, so $C_{m}$ is a correspondence of degree one. This implies that $\operatorname{deg}\left(T_{m}\right) \leq \operatorname{deg}(\pi)$, and the result follows, as $\operatorname{deg}\left(T_{m}\right)=\psi(m) \geq m+1$.

\section{References}

[1] G. Faltings, "Endlichkeitssätze für abelsche Varietäten über Zahlkörpern", Invent. Math. 73 (1983), 349-366.

[2] S. Lang, "Elliptic Functions, 2nd Edition", Springer-Verlag 1987.

[3] M. Raynaud, "Courbes sur une variété abélienne et points de torsion", Invent. Math. 71 (1983), 207-233. 
[4] K.A. Ribet, "Torsion points of abelian varieties in cyclotomic extensions", Appendix to: N.M. Katz, S. Lang, "Finiteness theorems in geometric class field theory", Enseign. Math. (2) 27 (1981), 285-319.

[5] J.-P. Serre, "Propriétés galoisiennes des points d'ordre fini des courbes elliptiques", Invent. Math. 15 (1972), 259-331.

Department of Mathematics, University of Stellenbosch, Stellenbosch 7600, South AFRICA.

E-mail address: fbreuer@sun.ac.za 